\title{
Communication-Based Influence Components Model
}

\author{
Brian Cugelman \\ University of Wolverhampton \\ Statistical Cybermetrics \\ Research Group \\ United Kingdom \\ b.cugelman@wlv.ac.uk
}

\author{
Prof Mike Thelwall \\ University of Wolverhampton \\ Statistical Cybermetrics \\ Research Group \\ United Kingdom \\ m.thelwall@wlv.ac.uk
}

\author{
Prof Phil Dawes \\ University of Wolverhampton \\ Wolverhampton Business School \\ United Kingdom \\ p.dawes@wlv.ac.uk
}

\begin{abstract}
This paper discusses problems faced by planners of real-world online behavioural change interventions who must select behavioural change frameworks from a variety of competing theories and taxonomies. As a solution, this paper examines approaches that isolate the components of behavioural influence and shows how these components can be placed within an adapted communication framework to aid the design and analysis of online behavioural change interventions. Finally, using this framework, a summary of behavioural change factors are presented from an analysis of 32 online interventions.
\end{abstract}

\section{Categories and Subject Descriptors}

H. Information systems H1. Models and principles H1.2. User/machine systems. Software psychology.

\section{General Terms}

Measurement, Design, Human Factors, Standardization, Theory.

\section{Keywords}

communication, social marketing, behaviour, internet, online, web

\section{Introduction}

To improve people's quality of life, numerous researchers and practitioners are developing and testing the efficacy of online health interventions. A few examples include interactive websites designed to help people increase their physical activity [1], reduce their binge drinking [2] and quit smoking [3]. Although there are many cases of effective online behavioural change interventions, it is not always clear why some online interventions have been shown to work, while others have not.

When considering possible explanations, across numerous webbased health interventions, there seems to be few clear-cut examples of theory A, behavioural change technique B, or persuasive strategy C. Rather, interventions normally blend concepts from different theories, while merging numerous factors that may exert influence in complex ways. This may explain why meta-analyses of web-based behavioural change interventions have shown that interventions can work online [4, 5]; however, they have not yet isolated the factors behind successful and unsuccessful interventions.

For example, consider an interactive website designed to influence users' behaviours that could place them at risk of contracting HIV/AIDS. Based on information entered about their lifestyle, this website may show users their risks, and then propose tailored solutions such as abstinence or practicing safe sex.
Assuming this intervention was successful to some degree, the factors that account for its efficacy may be attributed to source credibility, ingenious tailoring algorithms, argument quality, the vividness of imagery used, fear appeals or how audiences relate to the communication style. All or some of these factors may account for the intervention's effectiveness or ineffectiveness; however, when trying to describe interventions, there is no comprehensive way of conceptualizing the many factors, nor a universal taxonomy of influence effects appropriate to the design of online behavioural change interventions.

Without a comprehensive framework to describe online behavioural change interventions, researchers and developers face problems when analyzing prior interventions and planning future ones. As a solution, this paper presents a communication-based framework that accounts for a wide variety of influence factors that are always present in real-world communication contexts. This framework is the result of a meta-analysis of online behavioural change interventions; it has iteratively grown from a consolidation of influence systems into a coding scheme designed to account for a large number of behavioural change factors. This paper describes the theoretical basis for the coding frame, while the final section presents a summary of 32 online behavioural change interventions from 31 studies. Although this framework was developed for online interventions, it may have wider application to communications campaigns in general.

\section{Behavioural Influence Frameworks}

Across different psychological and behavioural change frameworks, there exists a complex blend of different classification systems, from different perspectives, with competing theories and influence techniques. Some are concerned with covert persuasion, and others, with overt techniques. Some are concerned with psychological constructs, while others, only with behaviour. One review of 42 frameworks described how some systems included taxonomies of discrete influence tactics; others clustered them around core psychological constructs; and the organizing principles ranged from a one dimensional system to a comprehensive list of 126 individual tactics [6].

For the sake of simplicity, in this paper, the term influence describes any approach aiming to change psychological faculties (such as attitudes), behaviours or both. An influence framework is any system that organizes multiple factors believed to modify people's psychology and/or behaviour. This paper is primarily concerned with behaviour, and the term behavioural influence interventions (BII) describes any intervention designed to improve peoples' lives, for example, by encouraging them to adopt healthy lifestyles, such as exercising more, eating healthier foods or quitting smoking. 


\subsection{Review of Influence Frameworks}

In order to assess whether the different influence frameworks fit together, this section reviews five influence frameworks from different disciplines. The purpose is to examine their organizing principles and assumptions; consequently, this brief review only addresses these systems as a whole, rather than individual tactics.

First, evidence-based behavioural medicine focuses on health promotion or disease prevention approaches, for which there is evidence of clinical efficacy or effectiveness [7]. Within this field, one initiative has used coders to extract theory-based behavioural change techniques from numerous health interventions. This has resulted in a comprehensive taxonomy of behavioural change techniques (BCTs). Instead of focusing on groupings, this initiative presents discrete techniques in a check-list format [8]. Having extracted BCTs from numerous theories, subsequent work has relied on coders to remap techniques to the underlying psychological constructs associated with behaviour, called behavioural determinants [9]. The organizing principle behind this approach is a comprehensive check-list of what works.

Second, as a general system to describe influence, Cialdini argues that there are thousands of compliance tactics; however, all these tactics impact just six psychological principles: reciprocity, commitment and consistency, social proof, liking, authority and scarcity [10]. Reciprocity is the human desire to repay another person in-kind. Consistency and commitment describe a person's desire to be consistent with past behaviour and how past commitments can be leveraged to influence future behaviour. Social proof describes peoples' tendency to take behavioural cues from their social context. Liking is the principle that people are more compliant with people they like. Authority describes how people are more likely to act on the advice of authority figures. Finally, scarcity describes how people assign more value to things that are less available. Hence, Cialdini organizes influence factors by broad psychological constructs; and within each construct, he outlines relevant moderating factors and influence techniques.

Third, CAPTOLOGY focuses on computers as persuasive technology. The founding book on the subject describes how technology can exert influence on users' psychology and behaviour. This system presents three broad groupings that describe how persuasive interaction may operate: as tools, media or social actors [11]. For example, people may be persuaded by technology while using it as a tool to complete tasks, such as receiving feedback or being guided through processes. They can be persuaded when using technology as media, such as being shown cause and effect relationships. Finally, as a social actor, technology can mimic attributes of human interaction; consequently, technology can express aspects of human influence. In summary, the CAPTOLOGY system classifies influence tactics according to how people use and/or interact with technology. This system has been extended with techniques grouped by primary task support, dialogue support, system credibility support and social support [12].

Fourth, the stages of change approach, also called the transtheoretical model, takes the position that all psychology disciplines do the same thing: they encourage people to progress through stages from pre-contemplation, to contemplation, to intention to act, to action, and finally, to maintenance; and during this process, people continually relapse before making progress. According to this system, all change happens according to ten processes, which are divided into two groups. The experiential processes include consciousness-raising, dramatic relief, selfreevaluation, environmental reevaluation and social liberation. Behavioural processes include self-liberation, helping relationships, counter-conditioning, reinforcement management and stimulus control. The factors that motivate people to move through stages are self-efficacy and decision balance (pros and cons) $[13,14]$. This system is organized around a model of how people progress through stages that are influenced by ten processes, two drivers of change, and many techniques that may affect any of these constructs.

Fifth, the field of social marketing adopts marketing practices and techniques, along with other approaches, to encourage population-wide behavioural change. The primary influence model is based on defining a 'product' which can be rendered more or less appealing by modifying its pros and cons. This is achieved by controlling the factors that may increase or decrease public demand: product, price, place and promotion [15]. The primary social marketing publication that most resembles an influence framework comes from the area of community-based social marketing. This approach begins with pre-campaign research aimed at discovering barriers and benefits associated with target behaviours. It then develops interventions and presents a framework of five techniques that have been proven to impact on behaviour: obtaining commitments, using prompts, appealing to norms, using communication and incentives. Across the five techniques, 31 further factors are presented that can impact on the implementation of the five [16]. This system is organized around a planning process that identifies and removes obstacles, develops incentives and employs motivation techniques.

Comparing the different influence frameworks, there are a number of noteworthy differences. Within the systems, influence techniques are arranged according to psychological principles, how people use/interact with technology, stages and processes of change, intervention planning processes, or a simple shopping list of what works. The use of theory differs considerably across these frameworks: the transtheoretical approach is both a theory and compilation of techniques; the remaining frameworks mix a wide number of theories to explain phenomena, while behavioural medicine draws on approaches that have been empirically shown to work, and places less emphasis on theory.

\subsection{Designing Real-World Interventions}

When designing real-world BII, practitioners are rarely confined by fixed theoretical frameworks. Normally, they blend theoretical constructs and techniques with practical, financial and political considerations. One review of the major theories used by behaviour change practitioners included the Health Belief Model, Stages of Change, Theory of Planned Behaviour, the Precaution Adoption Process Model, Social Cognitive Theory, Community Organization, Diffusion of Innovations and Communications Theory. The authors advised health campaigners to conduct research on their audiences and behavioural goals, then pick the theory or blend of theories that seem most suitable [17]. Likewise, social marketing interventions normally progress through early thinking, concept testing, revisions, pilot testing and further revisions before being considered for deployment. Once mature, an intervention is likely to contain elements of various theories, techniques and considerations. 
When blending features of various theories and practices, practitioners need to understand the comparative differences between influence frameworks, the way they group techniques, how they relate to theory and how one theory relates to another. They need to know which theories and psychological constructs are best represented by which influence frameworks, and which influence techniques 'belong' to which theories. Additionally, they need to consider numerous practical considerations not addressed by these frameworks, such as graphic design, target audience traits, social trends and institutional politics.

\section{Influence Components}

Traditional behavioural change theories hypothesise that BCTs (influencers) can influence psychological constructs (behavioural determinant), which in turn may influence behavioural outcomes. Figure 1 presents this three-stage model which is common across numerous behavioural change theories and was derived from a larger model in behavioural medicine [9].

Influencers $\rightarrow$ Behavioural $\rightarrow \begin{aligned} & \text { Behavioural } \\ & \text { Determinant }\end{aligned} \rightarrow$

Figure 1: Three-stage model of behavioural change (see Figure 2 for an extended model)

As an example of this model, consider Social Cognitive Theory which is built from the strong correlation between a person's selfefficacy and their ability to achieve a behavioural goal [18]. With this approach, a therapist helping a person quit smoking may use BCTs that help the smoker build confidence that they can quit smoking (influencer); this is designed to boost their self-efficacy (behavioural determinant), which would help them increase their odds of quitting smoking (behavioural outcome). For a second example, the Theory of Planned Behaviour posits that numerous techniques (influencers) can be deployed to influence three behavioural determinants: attitudes, perceived social norms, and self-efficacy, which impact on behavioural intent, that can motivate behaviour [19].

One meta-analysis used moderation analysis to show the effect sizes associated with particular BCTs, determinants and behavioural outcomes. The authors demonstrated the statistical associations between attitudinal arguments (BCT), attitude change (determinants) and condom use (behavioural outcomes); they also demonstrated correlations between self-management training (BCT), behavioural skills (determinant) and condom use (behavioural outcome) [20]. Having broken theory down to influence components, this meta-analysis allowed the researchers to go beyond showing that a given HIV/AIDS intervention could work. Instead, it provided insights into how they worked by showing relationships between influencers, behavioural determinants and both psychological or behavioural outcomes. Moreover, this investigation showed which packages of techniques and determinants were more or less effective.

\subsection{Influence Components Approaches}

Although the three-stage model appears clear-cut, practitioners and researchers frequently focus on influencers and behavioural determinants, regardless of the theories involved. For example, in one investigation of physical activity websites, 19 health promotion experts were unable to agree on which BCTs belonged to which theories; consequently, the researchers were forced to code each theory and technique separately [21].
Instead of focusing on the behavioural determinants associated within the major theories, some researchers prefer to extract the key constructs across numerous theories. In other words, instead of asking what is theorized to work, they prefer to ask which behavioural determinants and influencers have been shown to work. For the sake of convenience, these initiatives are called influence components approaches. The following passages review three influence components approaches.

First, the Behavioural Change Consortium brought together 15 US health behavioural change programs to assess the effectiveness of public wellbeing interventions. One of their efforts examined mediator variables (behavioural determinants) associated with successful behavioural change interventions [22].

Second, evidence-based behavioural medicine seeks to synthesize and extract core building blocks of influence across numerous theories and real-world interventions. As discussed above, one initiative following this approach, has developed a BCT taxonomy based on numerous real-world interventions [8]. This initiative also engaged numerous health professionals and researchers to synthesize key psychological constructs associated with behavioural influence. Similar to the Behavioural Change Consortium approach above, this initiative extracted 128 influence constructs from 33 theories and reduced them to 12 core behavioural determinants [23]. Successive work has used coders to reassociate BCTs with their respective behavioural determinants [9].

Third, the evidence-based kernels approach seeks to identify the key components of behavioural change interventions. Allegorically expressed, behavioural kernels are like the "active ingredients" in medications that are proven to work. Formally expressed, kernels have two defining features. First, they have been empirically proven to exert a reliable effect on one or more behaviours. Second, they are the fundamental units of influence, in that the removal of kernels from an intervention would render it unable to exert influence [24].

A chief limitation of these influence components approaches is a lack of recognition that numerous influence factors co-exist in real-world interventions. For example, real-world interventions may be confounded or enhanced by the social context, media, source perceptions, message encoding choices, audience traits, user feedback or competing campaigns. For example, source credibility can affect a social campaign's behavioural impact [25]. Audience's personal relevance towards an issue can moderate the effect of source credibility [26]. Encoding an intervention through time, as a single or sequential intervention, using foot-in-the-door or door-in-the-face techniques can also influence the strength of an intervention [27].

\subsection{Influence Components Model}

As already discussed, real-world BIIs are normally based on complex blends of different theories and techniques. When considering how the three-stage model may be formulated in realworld BIIs, Figure 2 presents a conceptual model of how the different influence components (presented in Figure 1) may interact. In this model, all interventions can be described according to the behavioural outcome, determinants and influencers, with popular groupings called packages. 


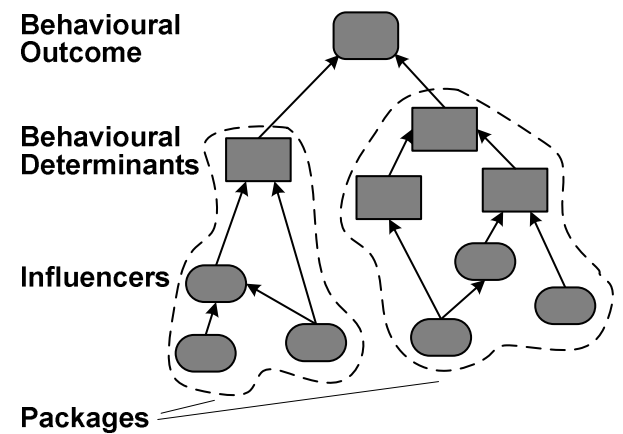

Figure 2: Conceptualization of influence components model

The first two components describe target audiences' psychology and behaviour. In this model, behavioural outcome describes the behaviour targeted by an intervention. Behavioural determinants describe the psychological constructs that are theorized to influence behaviour.

The next two components represent the building blocks of the BII: influencers and packages. Influencers describe components that have been empirically shown influence behaviour, and which are theorized to operate through behavioural determinants. As a broad term, influences describe overt and covert influence techniques; it can apply to traditional behavioural change techniques, persuasion, arguments or appeals. Within the communication process (described below), influence co-exists at several points along the communication process.

Packages are complex groupings that are commonly found across numerous interventions. Packages are likely to represent examples of 'best practices' or standard approaches where given components are frequently grouped together. For example, community based social marketing does not just advocate obtaining a commitment, but rather, packaging commitments by making them public (social norm determinant) or helping audiences see themselves as environmentally concerned (self identity determinant) [16]

\subsection{Complex Interactions in Real-World BIIs}

Although influence components approaches can simplify the analysis and design of interventions, these approaches also face considerable challenges. The ways complex influence packages interact is not always known. Evidence-based behavioural medicine recognizes that interventions are normally composed of distinct techniques; however, planners may not always understand which combinations may enhance effectiveness [8]. Conceptualization of how components interact is further complicated by the fact that depending on how planners regard relations between variables, each influence component may be described as an independent, mediator or moderator variable [28].

Popular behavioural change theories (such as the Theory of Planned Behaviour or Social Cognitive Theory) package psychological constructs and techniques that have been shown to operate well together; however, when psychological constructs are divorced from their theoretical grouping, it is possible that contradictory combinations could produce weaker rather than stronger effects.

\section{Interventions and Communication Theory}

Behavioural change interventions normally operate through multiple communication channels, such as the mass media, public events and direct marketing where the Internet is conceived as just one component of a larger campaign. For example, the Verb campaign aimed to encourage US tweens to be more physically active. This campaign was carried out through numerous communications channels, with a strong emphasis on long-term online relations [29]. Considering online BIIs may be conceived as part of mass marketing campaigns, as well as individually tailored interventions, this section reviews classical communication models to identify a framework suitable to online interventions.

\subsection{One-Way and Two-Way Communication Models}

Early one-way models divided the communication process into a series of steps, where a source sends a message through a medium to reach a recipient and exert influence upon them. We refer to these as one-way models. Presented in Figure 3, when targeting a single audience member, this type of one-way model is called the impersonal model (one-to-one). When applied to mass audiences, this is called the mass media model (one-to-many) [30, 31]. In general, one-way models are used to describe linear mass media relationship such as TV or newspapers where one source sends a message to many persons [32].

Perhaps the first one-way model was described over 2,000 years ago by Aristotle in his work on rhetoric, when he stated that communication was composed of a sender, message and recipient [33]. Likewise, communication has been describe as "Who, Says what, In which channel, To whom, With what effect" [34]. This framework was used by Carl Hovland who laid much of the groundwork for studying persuasive mass communication [35].

Applied to communications technology, the Shannon-Weaver model added a channel, signal, transmitter, receiver and noise [36]. Initially designed to describe signal to noise aspects of telecommunications, the Shannon-Weaver model inspired numerous models of human communication and brought standard terminology to communication studies; however, it also reinforced the tradition of one-way communication models [32].

By the mid 1950s, one-way models were modified to incorporate two aspects of communication that were previously ignored. The first was feedback; the second was a focus on meaning and the problems associated with transferring meaning as opposed to just messages [37]. Applied to interpersonal communication, feedback accounts for two-way models that have been called one-to-one $[30,31]$ or one-to-few [32], but are described here as one-withone to capture two-way communications or interaction.

One major criticism of one-way models was their disregard for individuals, their opinions, background, unique needs and capacities as intelligent autonomous beings. The adoption of oneway models has been cited as the reason why early research on mass persuasion produced so few findings [38]. Within the area of influence, perhaps one key difference between one and two-way models is the assumption that, in one-way transactions, a source sends a message to an audience that is influenced, while the twoway model describes an interactive process where the source and audience enter a bi-directional influence relationship. 


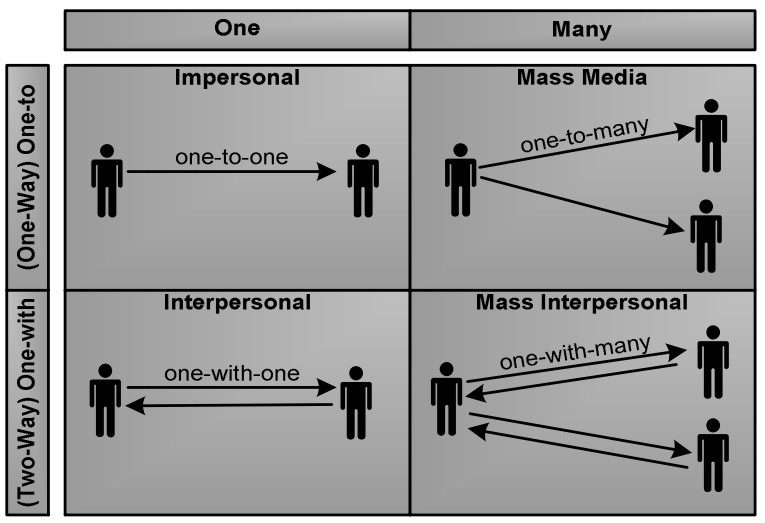

Figure 3: Four communication models

For the sake of describing models with or without feedback, the shorthand 'to' is used to describe one-way models. The term 'with' is used to describe two-way models based on feedback. Using this shorthand, the four models presented in Figure 3 describe three traditional models, and the mass interpersonal model. First, the impersonal model (one-to-one) describes the early one-way models that assumed influence flowed from source to audience. Applying this model to mass communications, the mass media model (one-to-many) describes tradition mass media such as TV, radio, newspapers and books. The interpersonal model (one-with-one) describes two-way communication between a source and audience, such as a discussion between two people, or a small group. The mass interpersonal model (one-with-many) is discussed below. In this paper many-with-many communication is not addressed, though it is frequently applied to computerbased communication and interaction [32].

Online, depending on a given perspective, communication may be described by a variety of models. The Internet is a network of computers where different communication applications operate, such as the world wide web, email, newsgroups, IRC. Depending on how applications are used, different communication models may be applied. From a macro perspective, the entire email system can be described as a many-with-many technology; from the viewpoint of an individual, it is one-with-many; and when looking at a particular transaction, it is one-with-one technology; but if that person is a spammer (sending unidirectional material), then their transaction could also be called one-to-many.

\subsection{Mass Interpersonal Communication}

The ecological model of behavioural change accounts for influence at numerous levels, such as the mass media, institutions, and interpersonal interactions [39]. For example, interpersonal influence can include one-with-one practices such as therapy, personal coaching or support services such as suicide hotlines. At the mass media level, therapy is sometimes applied to entire populations, such as national anti-smoking campaigns based on the stages of change approach [14].

The distinction between mass and interpersonal communication has been called a false dichotomy that is not based on significant differences, but is the result of separate research histories and institutional politics [32]. During the historical development of communication studies, mass and interpersonal communication developed separately by two academic groups that rarely interacted. Interpersonal communication emerged by psychologists and social psychologists in the 1930s. Mass communication was taken up by sociologists and political scientists interested in mass influence (propaganda). In the 1980s, computer-based communication was cited as an example of communication that could not be classified as either mass nor interpersonal [32]. Then in the 1990s, the Internet prompted researchers to argue that neither interpersonal nor mass media models explained Internet-based communication [31]. Today, this same lack of conceptual fit may explain why researchers argue that the Internet has blurred the distinctions between mass and interpersonal communication [40].

Many online BIIs conduct interpersonal communication with large numbers of users, resulting in communication that can be described from the micro-level as interpersonal, and from the macro-level as mass communication; however, when combined, as shown in Figure 3, this relationship is labelled mass interpersonal (one-with-many). Direct marketing provides an example of this model where a marketing department may engage large populations in personalized relations based on two-way communication through the exchange of print materials by mail. Online, the mass interpersonal model was described by evaluators of a healthy eating website who argued that counselling provided highly personal feedback, but could not reach large groups; mass media interventions could reach large groups, but with minimal personalization; however, they considered their web-based interventions to exist between the two extremes [41]

\section{Communication-Based Influence Components Model}

Real-world BIIs never happen in a vacuum; rather, they occur in complex social and communication context, where all aspects of the communication process may strengthen or weaken an intervention, and possibly produce unintended effects.

In seeking a communication framework applicable to online BIIs, a number of models were considered that could account for individually focused and mass media campaigns; communication and interaction; one and two-way communication; humancomputer and human-human transactions; while also accounting for overt and covert influence factors.

\subsection{Researching and Developing the Model}

In seeking a model that could meet these criteria, this review considered one and two-way communication models by Aristotle, Lasswell (1948), Shannon-Weaver (1949), Osgood and Schramm (1954), Berlo (1960) and DeFleur (1970). All the communication models reviewed contain a source, message and receiver. Most models included a media channel used to transmit the message. Some models include the process of encoding and decoding messages. A few included feedback in the form of two-way relationships. In addition, this review examined synthesis works on persuasive communication by Azjen (1992) and O'Keefe (2002). Both works grouped persuasive impacts by social context, source, media channel, message and receiver.

After considering the various criteria, the Osgood and Schramm (1954) model was adapted and placed within the context of persuasive effects described by Azjen (1992) and O'Keefe (2002). Combined with the influence components model, Figure 4 presents this model which is called the communication-based influence components model. 


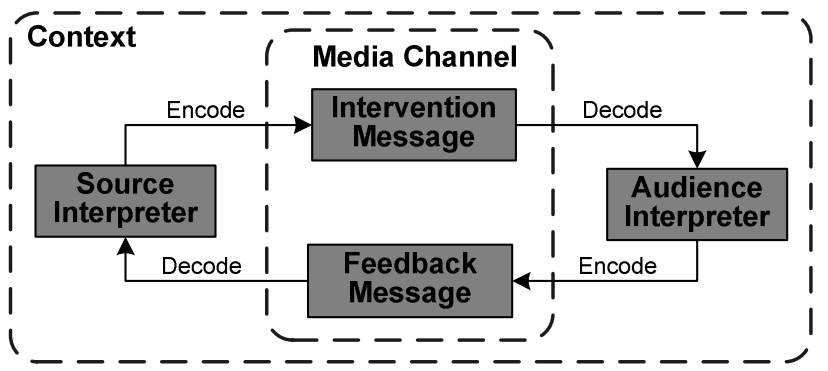

Figure 4: Communication-based influence components model

The reasons for selecting and modifying this model are as follows. First, the Osgood and Schramm (1954) model accounts for one and two-way communication, which is essential for personalization and tailoring.

Second, it describes interpersonal communication, but has also been applied to mass media communication [42].

Third, the model describes discrete actors in the communication process. The model has been criticized for giving equal weight to interacting actors because communication is often weighted in favour of one party [37]. However, this criticism can be addressed while adapting the model to the viewpoint of the intervention planner by relabelling the actors as the source and audience. By this distinction, the source sends the intervention message (the BII) to the audience who sends a feedback message back to the source.

Fourth, the original model describes how the communication may work by showing that each actor decodes, interprets and encodes messages. Although useful for explaining communication, these elements have been deemphasized in this model to stress encoding which is used to group influence factors and explain interaction.

Finally, the social context and media channels are added from work by Azjen (1992) and O'Keefe (2002) which explain influence components that may operate within these domains.

\subsection{Defining Model Elements}

This section describes how the influence components model (Figure 2) relates to the communication-based influence components model (Figure 4). Context represents the real world social and environmental factors that can impact on all aspects of a BIIs. Influencers include competition from alternative behaviours or sources; and social pressure to act or not act [43].

Source (and pseudo-source) interpreter defines the actor or system that decodes and interprets feedback messages from the audience before encoding an intervention message that is sent to the audience. In computer mediated environments, audiences frequently treat interactive systems as social actors [11, 44, 45], consequently, the term pseudo-source represents source factors that are misattributed to a computer system rather than the people/organization responsible for that system. An example would be attributing source credibility to a website because it looks credible [46] regardless of who operates it. Sources and pseudo-source influencers include credibility, attractiveness, likeability, similarity [26]; with additional influencers including reciprocation, commitments and authority [10]. Pseudo-source influencers may also include persuasive components of human communication: physical cues, psychological cues; language; social dynamics; social roles [11].
Source encoding describes the way a given BII may be expressed in time and space. Encoding represents the process of translating concepts into reality, and consequently, it crosses the threshold from source to media channel to the message (intervention). Encoding describes the effects associated with how an intervention is expressed as distinct from its substance. For example, an intervention may be encoded into a single or sequence of messages. It may be visually represented in numerous ways. It may be expressed through numerous media. Influencers include sequential request techniques such as the foot-in-door or door-in-face techniques [47]. In time and space, influencers can include tunnelling and reduction [11].

Media channel describes effects associated with different media, such as video, print, audio. The media channel encompasses the communication process through encoding, the intervention and feedback. Research on media influencers show that video can impact credibility and likeability more than audio; while written material is better remembered than video or audio [26]. At present, there appears to be a lack of consensus on whether applications such as email, the WWW or social networking constitute distinct media or are part of the Internet media.

Intervention message (source) represents the tangible communication containing influencers designed to impact behavioural determinant and ultimately behaviour. Influencers include BCTs, arguments and marketing offers. Examples of BCTs include those listed in a taxonomy of techniques [8], of which the top ten are listed in the following section. Factors which may modify intervention effectiveness include argument strength which is proposed to influence intervention impact, and is moderated by argument novelty, strength and relevance [38]. Also, marketing offers can be expressed as messages associated with services or products that aims to reduce barriers and increase incentives towards a target behaviour[16].

Audience interpreter describes the individual or population targeted to perform or adopt behavioural outcomes. For the communication cycle to continue, the audience must decode, interpret and act on a message. The intervention message will be designed to motivate behaviour by targeting audience's behavioural determinants [23]; these are presented in the audience interpreter paragraph in the following section.

Audience encoding describes the process whereby the audience provides feedback. For online BIIs, these are likely to be in the form of structured web forms or email. In the case of mass campaigns, feedback may be communicated indirectly and identified through market research about audiences, rather than directly expressed by audiences themselves. The primary influencers are providing incentives to send feedback, and reducing barriers that prevent people from sending feedback [16].

Feedback message (audience) describes information audiences send to the source. Whether used in individual or mass campaigns, tailored or timed interventions require user's feedback. Without feedback, it is impossible to design relevant or individually focused interventions. A wide number of behavioural influencers are derived from audience feedback. At the individual level, tailoring describes recommendations based on an assessment of individual traits. Personalization describes when interventions use personal information such as user's names. Adaptation/content matching is content that matches the personal traits and behavioural goal [48]. Stage matched interventions, 
based on the transtheoretical model, frequently begin with user assessment surveys to determine the appropriate intervention [14]. Kairos, which requires user and context feedback, describes the opportune time to communicate an intervention [11]. For mass campaigns, initial feedback (normally conducted through market research) is used to design behavioural proposals that are relevant, appealing and easy for target audiences to perform [16].

\subsection{Applying the Model}

To demonstrate how the communication-based influence components model can aid the analysis of interventions, this section presents descriptive statistics from an assessment of 31 research papers describing 32 online BIIs. The interventions in this section are derived from two searches of online BIIs from 1999-2008. Searches were made in Web of knowledge, PsycINFO, MEDLINE, PubMED and the Cochrane Library, producing 1,587 studies that were reduced to 31 qualifying papers which are listed at the end of this section. The full methods and statistical results will be presented in an upcoming paper, however, this section presents the distribution of influence components across the 32 online BIIs

For the social context, one intervention was national in scope, with $41 \%$ taking place in institutions, $9 \%$ in families, one targeting an individual and their friend, and $44 \%$ just targeting individuals. Within institutional contexts, it was common to enhance influence through appeal to social norms or engaging users in online group activities.

Of the media channels, all were conducted through websites (as this was a qualification criteria), $66 \%$ also used email, and 5 included minor human interaction by telephone, post or email.

Audience encoding was the starting point for most interventions, where users would provide feedback through baseline web surveys or when users subsequently logged-in, they were asked to provide data or undertake change stage assessments. Most systems used a number of audience feedback message mechanisms. Just one study used adoption/content matching, two used demographic matching; however, personalization was described in 38\% of studies with tailoring being the most widespread mechanism across $78 \%$ of the interventions. All the interventions that used personalization also used tailoring and across these interventions, $19 \%$ did not specify any feedback mechanisms.

Many interventions (65\%), did not describe source modifiers. Just one mentioned credibility, similarity was used across $12 \%$ of interventions, and attractiveness/design, across $21 \%$. For source encoding, the vast majority (78\%) of studies included multiple interactions, with just one based on the foot-in-the door technique. Three were conducted in a single session, with three not specified.

The intervention message containing the core influencers designed to impact behavioural determinants were coded from an updated version of the taxonomy of BCTs by Abraham and Michie (2008). Subject to grouping, the top ten most popular BCTs used across all 32 interventions included: Providing information on consequences of behaviour (78\%), Goal setting $(69 \%)$, Prompting self-monitoring of behaviour or outcomes (63\%), Providing feedback on performance (63\%), Providing instruction on how to perform the behaviour (63\%), Action planning (59\%), Providing normative information about others' approval or behaviour (44\%), Fear Arousal (38\%), Barrier
identification/Problem solving (34\%) and Providing information on where and when to perform the behaviour (34\%).

Finally, audience interpreter components contain behavioural determinants theorized to influence behaviour. Across all interventions, variables from an earlier taxonomy by Michie et al. (2005) included: Knowledge (100\%), Motivation and goals (Intentions) (88\%), Social influences (Norms) (75\%), Beliefs about consequences $(72 \%)$, Skills $(66 \%)$, Memory, attention and decision processes (59\%), Behavioural regulation (56\%), Emotion (34\%), Beliefs about capabilities (Self-efficacy) (31\%), Nature of the behaviours (28\%), Environmental context and resources $(25 \%)$ and Social-professional role and identity (13\%).

Studies in this analysis: Bersamin et al. (2007), Bewick et al. (2008), Bruning Brown et al. (2004), Celio et al. (2000), Chiauzzi et al. (2005), Cullen et al. (2008), Dunton et al. (2008), Gueguen et al. (2001), Hunter et al. (2008), Jacobi et al. (2007), Kim et al. (2006), Kosma et al. (2005), Kypri et al. (2004), Kypri et al. (2005), Lenert et al. (2004), Marshall et al. (2003), McConnon et al. (2007), McKay et al. (2001), Moore et al. (2005), Napolitano et al. (2003), Oenema et al. (2005), Patten et al. (2006), Petersen et al. (2008), Roberto et al. (2007), Severson et al. (2008), Strecher et al. (2004), Strom et al. (2000), Swartz et al. (2006), Tate et al. (2001), Verheijden et al. (2004) and Winett et al. (2007).

\section{Discussion and Conclusion}

The communication-based influence components model can serve as a useful framework to describe or design online interventions, whether they adopt interpersonal or mass media approaches, whether they use overt or covert tactics, and whether they are focused on human mediated communication or direct human computer interaction. Although this model has resulted from a meta-analysis of online BIIs, it may be applied to a wider range of interventions.

For practical applications, this model can also aid the design of social marketing campaigns that seek to engage citizens through the use mass marketing approaches as well as automated personal relationships. By providing long-term, tailored and personal support, mass interpersonal campaigns can be a valuable way to engage citizens in public health, environmental protection, safety and social development initiatives.

\section{References}

[1] Marshall, A. Leslie, E. Bauman, A. Marcus, B. and Owen, N. 2003. Print versus website physical activity programs A randomized trial. American Journal of Preventive Medicine. 25(2): p. 88-94.

[2] Moore, M. Soderquist, J. and Werch, C. 2005. Feasibility and Efficacy of a Binge Drinking Prevention Intervention for College Students Delivered via the Internet Versus Postal Mail. Journal of American College Health. 54(1): p. 38-44.

[3] Lenert, L. Munoz, R. Perez, J. and Bansod, A. 2004. Automated email messaging as a tool for improving quit rates in an internet smoking cessation intervention. Journal of the American Medical Informatics Association. 11(4): p. 235-240.

[4] Wantland, D. Portillo, C. Holzemer, W. L. Slaughter, R. and McGhee, E. 2004. The effectiveness of web-based vs. non-webbased interventions: a meta-analysis of behavioural change outcomes. Journal of Medical Internet Research. 6(4).

[5] Portnoy, D. Scott-Sheldon, L. Johnson, B. and Carey, M. 2008. Computer-delivered interventions for health promotion and behavioral risk reduction: A meta-analysis of 75 randomized controlled trials, 1988-2007. Preventive Medicine. 47(1): p. 316. 
[6] Rhoads, K. 2002 An introduction to social influence. 8 December 2008]; Available from:

http://www.workingpsychology.com/intro.html.

[7] Davidson, K. Goldstein, M. Kaplan, R. Kaufmann, P. Knatterud, G. Orleans, C. Spring, B. Trudeau, K. and Whitlock, E. 2003. Evidence-Based Behavioral Medicine: What Is It and How Do We Achieve It? Annals of Behavioral Medicine. 26(3): p. 161171.

[8] Abraham, C. and Michie, S. 2008. A taxonomy of behavior change techniques used in interventions. Health Psychology. 27(3): p. 379-387.

[9] Michie, S. Johnston, M. Francis, J. Hardeman, W. and Eccles, M. 2008. From Theory to Intervention: Mapping Theoretically Derived Behavioural Determinants to Behaviour Change. Applied Psychology. 57(4): p. 660-680.

[10] Cialdini, R. 2008. Influence: science and practice. 5th ed, Boston: Pearson/Allyn and Bacon.

[11] Fogg, B. J. 2003. Persuasive technology: using computers to change what we think and do, San Francisco: Morgan Kaufmann Publishers.

[12] Oinas-Kukkonen, H. and Harjumaa, M. 2008. A Systematic Framework for Designing and Evaluating Persuasive Systems, in Lecture Notes in Computer Science, Springer Berlin / Heidelberg. p. 164.

[13] Prochaska, J. and Norcross, J. 2001. Stages of Change. Psychotherapy. 38(4): p. 443-448.

[14] Prochaska, J. Norcross, J. and DiClemente, C. 1995. Changing for Good: A Revolutionary Six-Stage Program for Overcoming Bad Habits and Moving Your Life Positively Forward: Collins

[15] Kotler, P. and Roberto, E. 1989. Social Marketing, New York: The Free Press.

[16] McKenzie-Mohr, D. and Smith, W. 1999. Fostering Sustainable Behavior--An Introduction to Community-Based Social Marketing, Gabriola Island, Canada: New Society Publishers.

[17] Glanz, K. Rimer, B. and Sharyn, D. 2005 Theory at a Glance: A Guide for Health Promotion Practice, U.S. Department of Health and Human Services, The National Cancer Institute.

[18] Bandura, A. 1982. Self-efficacy mechanism in human agency. American Psychologist. 37(2): p. 122-147.

[19] Ajzen, I. 1991. The theory of planned behavior. Organizational Behavior and Human Decision Processes. 50(2): p. 179-211.

[20] Albarracin, D. Gillette, J. Earl, A. Glasman, L. Durantini, M. and Ho, M. 2005. A Test of Major Assumptions About Behavior Change: A Comprehensive Look at the Effects of Passive and Active HIV-Prevention Interventions Since the Beginning of the Epidemic. Psychological Bulletin. 131(6): p. 856-897.

[21] Doshi, A. Patrick, K. Sallis, J. and Calfas, K. 2003. Evaluation of physical activity web sites for use of behavior change theories. Annals of Behavioral Medicine. 25(2): p. 105-111.

[22] Ory, M. Jordan, P. and Bazzarre, T. 2002 The Behavior Change Consortium: setting the stage for a new century of health behavior-change research, Oxford Univ Press. p. 500-511.

[23] Michie, S. Johnston, M. Abraham, C. Lawton, R. Parker, D. and Walker, A. 2005. Making psychological theory useful for implementing evidence based practice: a consensus approach. Quality and Safety in Health Care. 14(1): p. 26-33.

[24] Embry, D. and Biglan, A. 2008. Evidence-based Kernels: Fundamental Units of Behavioral Influence. Clinical Child and Family Psychology Review. 11(3).

[25] Kotler, P. and Zaltman, G. 1971. Social Marketing: An Approach to Planned Social Change. Journal of Marketing. $35(3,2)$.

[26] O'Keefe, D. 2002. Persuasion: Theory and Research, London: Sage Publications, Inc.
[27] Fern, F. Monroe, K. and Avila, R. 1986. Effectiveness of multiple request strategies: A synthesis of research results. Journal of Marketing Research. 23(2): p. 144-152.

[28] Frazier, P. Tix, A. and Barron, K. 2004. Testing Moderator and Mediator Effects in Counseling Psychology Research. Journal of Counseling Psychology. 51(1): p. 115-134.

[29] Huhman, M. 2008. New Media and the VERB Campaign: Tools to Motivate Tweens to be Physically Active. Cases in Public Health Communication \& Marketing. 2: p. 126-139.

[30] Hoffman, D. and Novak, T. 1996. Marketing in Hypermedia Computer-Mediated Environments: Conceptual Foundations. Journal of Marketing. 60: p. 50-68.

[31] O'Sullivan, P. 1999. Bridging the Mass-Interpersonal Divide Synthesis Scholarship in HCR. Human Communication Research. 25(4): p. 569-588.

[32] Reardon, K. and Rogers, E. 1988. Interpersonal versus mass media communications: a false dichotomy. Human Communications Research. 15(2): p. 284-303.

[33] Lawson-Tancred, H. 1992. Aristotle--The Art of Rhetoric Penguin Classics.

[34] Lasswell, H. 1948. The structure and function of communication in society. The Communication of Ideas: p. 37-51.

[35] Griffin, E. 2008. A first look at communication theory. 7 ed, New York: McGraw-Hill.

[36] Shannon, C. and Weaver, W. 1948. A mathematical theory of communications. Bell System Technical Journal. 27(2): p. 632656.

[37] McQuail, D. and Windahl, S. 1993. Communication Models for the Study of Mass Communications. 2nd ed, New York: Longman.

[38] Ajzen, I. 1992. Persuasive communication theory in social psychology: A historical perspective, in Influencing Human Behavior: theory and applications in recreation, tourism, and natural resources management, Manfredo, J., Editor, Sagamore Publishing: Illinois. p. 1-16.

[39] McLeroy, K. Bibeau, D. Steckler, A. and Glanz, K. 1988. An Ecological Perspective on Health Promotion Programs. Health Education \& Behavior. 15(4): p. 351-377.

[40] Abroms, L. and Maibach, E. 2008. The Effectiveness of Mass Communication to Change Public Behavior. Annual Review of Public Health. 29: p. 219.

[41] Oenema, A. and Brug, J. 2003. Feedback strategies to raise awareness of personal dietary intake: results of a randomized controlled trial. Preventive Medicine. 36(4): p. 429-439.

[42] Schramm, W. 1955. How Communications Works, in Mass Media \& Society, Wells, A. and Hakanen, E., Editors, Ablex Pub.

[43] Andreasen, A. 2006. Social marketing in the 21st century, Thousand Oaks: Sage Publications, Inc.

[44] Reeves, B. and Nass, C. 2003. The media equation: how people treat computers, television and new media like real people and places: University of Chicago Press; New Ed edition.

[45] Fogg, B. J. and Tseng, H. 1999 The elements of computer credibility, in CHI 99: Pittsburgh, USA.

[46] Cugelman, B. Thelwall, M. and Dawes, P. Website Credibility, Active Trust and Behavioural Intent. in Persuasive 2008, LNCS 5033. 2008. Berlin, Heidelberg: Springer-Verlag.

[47] Dillard, J. 1991. The Current Status of Research on SequentialRequest Compliance Techniques. Personality and Social Psychology Bulletin. 17(3): p. 283-288.

[48] Lustria, M. Cortese, J. Noar, S. and Glueckauf, R. 2008. Computer-tailored health interventions delivered over the web: Review and analysis of key components. Patient Education and Counseling. 